\title{
Geometric Least Square Models for Deriving [0, 1]-Valued Interval Weights from Interval Fuzzy Preference Relations Based on Multiplicative Transitivity
}

\author{
Xuan Yang ${ }^{1,2}$ and Zhou-Jing Wang ${ }^{3}$ \\ ${ }^{1}$ School of Accounting, Zhongnan University of Economics and Law, Wuhan, Hubei 430073, China \\ ${ }^{2}$ School of Accounting, Zhejiang University of Finance \& Economics, Hangzhou, Zhejiang 310018, China \\ ${ }^{3}$ School of Information, Zhejiang University of Finance \& Economics, Hangzhou, Zhejiang 310018, China \\ Correspondence should be addressed to Zhou-Jing Wang; wangzj@xmu.edu.cn
}

Received 11 November 2014; Revised 6 June 2015; Accepted 8 June 2015

Academic Editor: Roman Lewandowski

Copyright ( 2015 X. Yang and Z.-J. Wang. This is an open access article distributed under the Creative Commons Attribution License, which permits unrestricted use, distribution, and reproduction in any medium, provided the original work is properly cited.

\begin{abstract}
This paper presents a geometric least square framework for deriving $[0,1]$-valued interval weights from interval fuzzy preference relations. By analyzing the relationship among $[0,1]$-valued interval weights, multiplicatively consistent interval judgments, and planes, a geometric least square model is developed to derive a normalized $[0,1]$-valued interval weight vector from an interval fuzzy preference relation. Based on the difference ratio between two interval fuzzy preference relations, a geometric average difference ratio between one interval fuzzy preference relation and the others is defined and employed to determine the relative importance weights for individual interval fuzzy preference relations. A geometric least square based approach is further put forward for solving group decision making problems. An individual decision numerical example and a group decision making problem with the selection of enterprise resource planning software products are furnished to illustrate the effectiveness and applicability of the proposed models.
\end{abstract}

\section{Introduction}

The preference relation is a common framework for expressing decision-makers' (DMs') pairwise comparison results in multicriteria decision making (MCDM). One widely used preference relation takes the form of the multiplicative preference relation, which was introduced by Saaty [1] to structure DMs' pairwise comparison ratios in the analytic hierarchy process (AHP). Another popularly used preference relation takes the form of a fuzzy preference relation (also called a reciprocal preference relation $[2,3]) R=\left(r_{i j}\right)_{n \times n}$ whose element $r_{i j}$ denotes the fuzzy preference degree of the object $i$ over $j$ and satisfies $0 \leq r_{i j} \leq 1, r_{i i}=0.5$, and the additive reciprocal property of $r_{i j}+r_{j i}=1$. Over the last three decades, fuzzy preference relations have been extensively studied [4] and the fuzzy AHP has been widely applied to various MCDM problems such as the green port evaluation [5] and the location selection [6], to name a few.

All of judgments in a fuzzy preference relation are characterized by crisp values. However, in many real-world situations, DMs' subjective judgments may be bounded between lower and upper bounds due to complexity and indeterminacy of decision problems. Therefore, the concept of interval fuzzy preference relations (IFPRs) is introduced by $\mathrm{Xu}$ [7] to describe imprecise and uncertain judgment information, and an increasing research interest has been concentrating on employing IFPRs to help DMs make their decision analyses.

An important research topic for MCDM with preference information is to derive priority weight vectors from preference relations. As preference information contains two kinds of uncertainty, that is, DM's judgments and inconsistency 
among comparisons, the derived priority weights should be $[0,1]$-valued interval weights (or called interval probabilities [8-10]). Different methods have been developed to derive $[0,1]$-valued interval weights from IFPRs. Xu and Chen [11] define additively consistent IFPRs and multiplicatively consistent IFPRs from the viewpoint of the feasible regions and develop two linear-programming-based approaches for obtaining $[0,1]$-valued interval weights. Based on $\mathrm{Xu}$ and Chen's multiplicative consistency, Genç et al. [12] propose a formula to determine a $[0,1]$-valued interval weight vector of an IFPR, in which the original IFPR is converted into the one with multiplicative consistency. They also show that the derived $[0,1]$-valued interval weight vector is the same as the result obtained by the approach given in $\mathrm{Xu}$ and Chen [11]. Lan et al. [13] put forward an exchange method between additively consistent IFPRs and multiplicatively consistent IFPRs and devise a parametric algorithm to obtain $[0,1]$-valued interval weights by converting a multiplicatively consistent IFPR into an additively consistent IFPR. Xia and Xu [14] establish two parametric programming models to generate $[0,1]$-valued interval weights of an IFPR. From the viewpoint of interval arithmetic, Wang and Li [15] define additively consistent IFPRs, multiplicatively consistent IFPRs, and weakly transitive IFPRs and design two goal programs to generate $[0,1]$-valued interval weights for individual and collective decisions.

The literature review indicates that among the priority methods mentioned above for IFPRs, most of them are developed according to the feasible-region-based consistency definitions and are only applicable to one IFPR. Although Wang and Li's approach [15] may be used to derive a group $[0,1]$-valued interval weight vector directly from individual IFPRs, it requires the importance weights of DMs or the relative weights of individual IFPRs to be known. It is very hard to assign the subjective weights to DMs in some group decision situations, such as the group decision making problem with a hierarchical structure in Section 5. On the other hand, so far little research has been found on employing the idea of geometric least squares to generate priority weights from IFPRs and determining the relative weights of individual IFPRs in group decision situations. In this paper, we develop a geometric least square model to derive $[0,1]$-valued interval weights from an IFPR. To measure the relative importance of individual IFPRs, the difference ratio between any two IFPRs is introduced to define the geometric average difference ratio between one IFPR and the others. A geometric least square based approach is further developed for solving group decision making problems with unknown DMs' weights.

The rest of the paper is set out as follows. Section 2 reviews some basic notions related to fuzzy preference relations and multiplicatively consistent IFPRs. A geometric least square model is established for deriving a $[0,1]$-valued interval weight vector from an IFPR in Section 3. Section 4 puts forward a method for determining the relative importance weights of individual IFPRs and develops a geometric least square based approach for deriving a group priority weigh vector directly from individual IFPRs. Section 5 provides a case study on the enterprise resource planning software product selection problem. Section 6 draws the main conclusions.

\section{Preliminaries}

For an MCDM problem, let $X=\left\{x_{1}, x_{2}, \ldots, x_{n}\right\}$ be an alternative set and let $R=\left(r_{i j}\right)_{n \times n}$ be a pairwise comparison matrix on $X$; if $R$ satisfies

$$
\begin{aligned}
0 & \leq r_{i j} \leq 1, \\
r_{i j}+r_{j i} & =1, \\
r_{i i} & =0.5,
\end{aligned}
$$

$$
\forall i, j=1,2, \ldots, n
$$

then $R$ is called a fuzzy preference relation.

The element $r_{i j}$ in $R$ gives a $[0,1]$-valued importance or fuzzy preference degree of $x_{i}$ over $x_{j}$. As the additive reciprocal property of $r_{i j}+r_{j i}=1$, the larger the value of $r_{i j}$, the stronger the preference ratio $r_{i j} / r_{j i}$ of $x_{i}$ over $x_{j}$. If $r_{i j}>0.5$, then $r_{i j} / r_{j i}>1$ and $x_{i}$ is preferred to $x_{j}$ with the ratio $r_{i j} / r_{j i}$. If $r_{i j}<0.5$, then $r_{i j} / r_{j i}<1$ and $x_{i}$ is nonpreferred to $x_{j}$ with the ratio $r_{i j} / r_{j i}$. In particular, if $r_{i j}=0.5$, then $r_{i j} / r_{j i}=1$, indicating that $x_{i}$ and $x_{j}$ are indifferent.

Definition 1 (see [16]). Let $R=\left(r_{i j}\right)_{n \times n}$ be a fuzzy preference relation with $0<r_{i j}<1, \forall i, j=1,2, \ldots, n$. $R$ is said to have multiplicative consistency, if it satisfies transitivity condition:

$$
\frac{r_{i k}}{r_{k i}}=\frac{r_{i j}}{r_{j i}} \frac{r_{j k}}{r_{k j}}, \quad \forall i, j, k=1,2, \ldots, n .
$$

It is obvious that (2) is equivalent to any of the following equations:

$$
\begin{gathered}
r_{i j} r_{j k} r_{k i}=r_{i k} r_{k j} r_{j i}, \quad \forall i, j, k=1,2, \ldots, n . \\
\frac{r_{i j}}{r_{j i}} \frac{r_{j k}}{r_{k j}} \frac{r_{k i}}{r_{i k}}=\frac{r_{i k}}{r_{k i}} \frac{r_{k j}}{r_{j k}} \frac{r_{j i}}{r_{i j}}, \quad \forall i, j, k=1,2, \ldots, n .
\end{gathered}
$$

With increasing complexity and indeterminacy in many decision problems, it is often difficult for a DM to furnish crisp preference degrees. To better model vague and uncertain DM's judgments, $\mathrm{Xu}$ [7] introduces the concept of IFPRs.

Definition 2 (see [7]). An IFPR $\bar{R}$ on $X$ is characterized by an interval-valued pairwise comparison matrix $\bar{R}=\left(\bar{r}_{i j}\right)_{n \times n}$ satisfying the following condition:

$$
\begin{aligned}
\bar{r}_{i j} & =\left[r_{i j}^{-}, r_{i j}^{+}\right], \\
0 & \leq r_{i j}^{-} \leq r_{i j}^{+} \leq 1, \\
r_{i j}^{-}+r_{j i}^{+} & =1, \\
r_{i i}^{-} & =r_{i i}^{+}=0.5,
\end{aligned}
$$

$$
\forall i, j=1,2, \ldots, n
$$


where $\bar{r}_{i j}$ denotes an interval importance or preference degree of $x_{i}$ over $x_{j}$.

Given two interval numbers $\bar{a}_{1}=\left[a_{1}^{-}, a_{1}^{+}\right]$and $\bar{a}_{2}=$ $\left[a_{2}^{-}, a_{2}^{+}\right]$, their arithmetic operation laws are summarized as follows.

(1) Addition: $\bar{a}_{1} \oplus \bar{a}_{2}=\left[a_{1}^{-}+a_{2}^{-}, a_{1}^{+}+a_{2}^{+}\right]$.

(2) Subtraction: $\bar{a}_{1}-\bar{a}_{2}=\left[a_{1}^{-}-a_{2}^{+}, a_{1}^{+}-a_{2}^{-}\right]$.

(3) Multiplication: $\bar{a}_{1} \otimes \bar{a}_{2}=\left[a_{1}^{-} a_{2}^{-}, a_{1}^{+} a_{2}^{+}\right]$, where $a_{1}^{-}>$ $0, a_{2}^{-}>0$.

(4) Division: $\bar{a}_{1} / \bar{a}_{2}=\left[a_{1}^{-} / a_{2}^{+}, a_{1}^{+} / a_{2}^{-}\right]$, where $a_{1}^{-}>0, a_{2}^{-}>$ 0.

Based on interval arithmetic, Wang and Li [15] introduce the multiplicative transitivity to define consistent IFPRs.

Definition 3 (see [15]). Let $\bar{R}=\left(\bar{r}_{i j}\right)_{n \times n}=\left(\left[r_{i j}^{-}, r_{i j}^{+}\right]\right)_{n \times n}$ be an IFPR with $0<r_{i j}^{-} \leq r_{i j}^{+}<1(i, j=1,2, \ldots, n) . \bar{R}$ is said to have multiplicative consistency, if $\bar{R}$ satisfies

$$
\frac{\bar{r}_{i j}}{\bar{r}_{j i}} \otimes \frac{\bar{r}_{j k}}{\bar{r}_{k j}} \otimes \frac{\bar{r}_{k i}}{\bar{r}_{i k}}=\frac{\bar{r}_{i k}}{\bar{r}_{k i}} \otimes \frac{\bar{r}_{k j}}{\bar{r}_{j k}} \otimes \frac{\bar{r}_{j i}}{\bar{r}_{i j}},
$$

$$
\forall i, j, k=1,2, \ldots, n \text {. }
$$

Let $\bar{\omega}_{i}=\left[\omega_{i}^{-}, \omega_{i}^{+}\right]$be the priority weight of the alternative $x_{i}$ for $i=1,2, \ldots, n$; then the ratio-based interval preference intensity of the alternative $x_{i}$ over $x_{j}$ can be determined as $\bar{\omega}_{i} / \bar{\omega}_{j}$. As per interval arithmetic, one can obtain $\bar{\omega}_{i} / \bar{\omega}_{j}=$ $\left[\omega_{i}^{-} / \omega_{j}^{+}, \omega_{i}^{+} / \omega_{j}^{-}\right]$. The ratio-based preference intensities are based on the positive real line with the neutral value 1 denoting the indifference between two alternatives. On the other hand, $[0,1]$-valued interval judgments in IFPRs are based on the bipolar unit interval scale having the neutral element 0.5 . There exists a relation between ratio-based judgment $a$ and $[0,1]$-valued judgment $r$; that is, $r=a /(1+a)$, implying that $\left[\left(\omega_{i}^{-} / \omega_{j}^{+}\right) /\left(1+\omega_{i}^{-} / \omega_{j}^{+}\right),\left(\omega_{i}^{+} / \omega_{j}^{-}\right) /\left(1+\omega_{i}^{+} / \omega_{j}^{-}\right)\right]=$ $\left[\omega_{i}^{-} /\left(\omega_{i}^{-}+\omega_{j}^{+}\right), \omega_{i}^{+} /\left(\omega_{i}^{+}+\omega_{j}^{-}\right)\right]$is the $[0,1]$-valued interval preference intensity of the alternative $x_{i}$ over $x_{j}$. Based on this idea, the following transformation formula is proposed by Wang and $\mathrm{Li}[15]$ to convert $[0,1]$-valued interval weight vector $\bar{\omega}$ into multiplicatively consistent IFPR $\bar{R}=\left(\bar{r}_{i j}\right)_{n \times n}$, where

$$
\bar{r}_{i j}=\left[r_{i j}^{-}, r_{i j}^{+}\right]= \begin{cases}{[0.5,0.5]} & i=j \\ {\left[\frac{\omega_{i}^{-}}{\omega_{i}^{-}+\omega_{j}^{+}}, \frac{\omega_{i}^{+}}{\omega_{i}^{+}+\omega_{j}^{-}}\right]} & i \neq j\end{cases}
$$

and $\bar{\omega}=\left(\bar{\omega}_{1}, \bar{\omega}_{2}, \ldots, \bar{\omega}_{n}\right)^{T}=\left(\left[\omega_{1}^{-}, \omega_{1}^{+}\right],\left[\omega_{2}^{-}, \omega_{2}^{+}\right], \ldots,\left[\omega_{n}^{-}\right.\right.$, $\left.\left.\omega_{n}^{+}\right]\right)^{T}$ is a normalized $[0,1]$-valued interval weight vector such that

$$
0<\omega_{i}^{-} \leq \omega_{i}^{+} \leq 1
$$

$$
\begin{gathered}
\sum_{j=1, j \neq i}^{n} \omega_{j}^{-}+\omega_{i}^{+} \leq 1, \\
\omega_{i}^{-}+\sum_{j=1, j \neq i}^{n} \omega_{j}^{+} \geq 1
\end{gathered}
$$

$$
i=1,2, \ldots, n \text {. }
$$

Let $\bar{\omega}_{i}=\left[\omega_{i}^{-}, \omega_{i}^{+}\right]$and $\bar{\omega}_{j}=\left[\omega_{j}^{-}, \omega_{j}^{+}\right]$be any two $[0,1]-$ valued interval weights; then, the following possibility degree formula is defined [11] and employed to compare them:

$$
\begin{aligned}
& P\left(\bar{\omega}_{i} \geq \bar{\omega}_{j}\right) \\
& \quad=\max \left\{1-\max \left(\frac{\omega_{j}^{+}-\omega_{i}^{-}}{\omega_{i}^{+}-\omega_{i}^{-}+\omega_{j}^{+}-\omega_{j}^{-}}, 0\right), 0\right\} .
\end{aligned}
$$

\section{A Geometric Least Square Model for an IFPR}

This section develops a geometric least square model to derive normalized $[0,1]$-valued interval weights from an IFPR.

As per (6), for IFPR $\bar{R}=\left(\bar{r}_{i j}\right)_{n \times n}=\left(\left[r_{i j}^{-}, r_{i j}^{+}\right]\right)_{n \times n}$ with $0<$ $r_{i j}^{-} \leq r_{i j}^{+}<1(i, j=1,2, \ldots, n)$, if there exists normalized $[0,1]$-valued interval weight vector $\bar{\omega}=\left(\bar{\omega}_{1}, \bar{\omega}_{2}, \ldots, \bar{\omega}_{n}\right)^{T}=$ $\left(\left[\omega_{1}^{-}, \omega_{1}^{+}\right],\left[\omega_{2}^{-}, \omega_{2}^{+}\right], \ldots,\left[\omega_{n}^{-}, \omega_{n}^{+}\right]\right)^{T}$ satisfying

$$
\begin{aligned}
& r_{i j}^{-}=\frac{\omega_{i}^{-}}{\omega_{i}^{-}+\omega_{j}^{+}}, \\
& r_{i j}^{+}=\frac{\omega_{i}^{+}}{\omega_{j}^{-}+\omega_{i}^{+}},
\end{aligned}
$$

$\forall i \neq j=1,2, \ldots, n$,

then $\bar{R}$ has multiplicative consistency.

As $r_{i j}^{-}+r_{j i}^{+}=1$ for all $i, j=1,2, \ldots, n$, it follows from (9) that

$$
\begin{aligned}
& \frac{r_{i j}^{-}}{r_{j i}^{+}}=\frac{\omega_{i}^{-}}{\omega_{j}^{+}}, \\
& \frac{r_{i j}^{+}}{r_{j i}^{-}}=\frac{\omega_{i}^{+}}{\omega_{j}^{-}},
\end{aligned}
$$


Clearly, (10) can be equivalently converted into

$$
\begin{aligned}
& r_{i j}^{-} \omega_{j}^{+}-r_{j i}^{+} \omega_{i}^{-}=0, \\
& r_{i j}^{+} \omega_{j}^{-}-r_{j i}^{-} \omega_{i}^{+}=0,
\end{aligned}
$$

$$
\forall i \neq j=1,2, \ldots, n
$$

According to the theory of analytical geometry, we can view $r_{i j}^{-} \omega_{j}^{+}-r_{j i}^{+} \omega_{i}^{-}=0$ and $r_{i j}^{+} \omega_{j}^{-}-r_{j i}^{-} \omega_{i}^{+}=0(i \neq j=$ $1,2, \ldots, n)$ as a number of planes. Thus, the corresponding normalized $[0,1]$-valued interval weight vector $\bar{\omega}=\left(\bar{\omega}_{1}, \bar{\omega}_{2}\right.$, $\left.\ldots, \bar{\omega}_{n}\right)^{T}=\left(\left[\omega_{1}^{-}, \omega_{1}^{+}\right],\left[\omega_{2}^{-}, \omega_{2}^{+}\right], \ldots,\left[\omega_{n}^{-}, \omega_{n}^{+}\right]\right)^{T}$ can be seen as an intersection point of these planes.

On the other hand, (11) holds for multiplicatively consistent IFPRs. In the real-life decision situations, IFPRs furnished by DMs are often inconsistent and may not be denoted by (11). In other words, the planes $r_{i j}^{-} \omega_{j}^{+}-r_{j i}^{+} \omega_{i}^{-}=0$ and $r_{i j}^{+} \omega_{j}^{-}-r_{j i}^{-} \omega_{i}^{+}=0(i \neq j=1,2, \ldots, n)$ have no unified intersection point. In this case, one has to seek $2 n$-space point $\left(\omega_{1}^{-}, \omega_{1}^{+}, \omega_{2}^{-}, \omega_{2}^{+}, \ldots, \omega_{n}^{-}, \omega_{n}^{+}\right)^{T}$ satisfying (7) as close to each plane as possible.

Let

$$
\begin{aligned}
& d_{i j}^{(1)}=\frac{\left|r_{i j}^{-} \omega_{j}^{+}-r_{j i}^{+} \omega_{i}^{-}\right|}{\sqrt{\left(r_{i j}^{-}\right)^{2}+\left(r_{j i}^{+}\right)^{2}}}, \\
& d_{i j}^{(2)}=\frac{\left|r_{i j}^{+} \omega_{j}^{-}-r_{j i}^{-} \omega_{i}^{+}\right|}{\sqrt{\left(r_{i j}^{+}\right)^{2}+\left(r_{j i}^{-}\right)^{2}}}
\end{aligned}
$$

$$
\forall i \neq j=1,2, \ldots, n
$$

where $d_{i j}^{(1)}$ and $d_{i j}^{(2)}$ denote the distances from the point $\left(\omega_{1}^{-}\right.$, $\left.\omega_{1}^{+}, \omega_{2}^{-}, \omega_{2}^{+}, \ldots, \omega_{n}^{-}, \omega_{n}^{+}\right)^{T}$ to the planes $r_{i j}^{-} \omega_{j}^{+}-r_{j i}^{+} \omega_{i}^{-}=0$ and $r_{i j}^{+} \omega_{j}^{-}-r_{j i}^{-} \omega_{i}^{+}=0$, respectively.
Obviously, the smaller the sum of the values of the distances $d_{i j}^{(1)}$ and $d_{i j}^{(2)}$ is, the closer the $\bar{R}$ is to a multiplicatively consistent IFPR. Therefore, reasonable point $\left(\omega_{1}^{-}, \omega_{1}^{+}, \omega_{2}^{-}, \omega_{2}^{+}, \ldots, \omega_{n}^{-}, \omega_{n}^{+}\right)^{T}$ can be determined by solving the following geometric least square optimization model:

$$
\begin{aligned}
& \min \quad J=\sum_{i=1}^{n} \sum_{j \neq i, j=1}^{n}\left(\frac{\left(r_{i j}^{-} \omega_{j}^{+}-r_{j i}^{+} \omega_{i}^{-}\right)^{2}}{\left(r_{i j}^{-}\right)^{2}+\left(r_{j i}^{+}\right)^{2}}+\frac{\left(r_{i j}^{+} \omega_{j}^{-}-r_{j i}^{-} \omega_{i}^{+}\right)^{2}}{\left(r_{i j}^{+}\right)^{2}+\left(r_{j i}^{-}\right)^{2}}\right) \\
& \text { s.t. }\left\{\begin{array}{l}
\omega_{i}^{+}+\sum_{j=1, j \neq i}^{n} \omega_{j}^{-} \leq 1, \quad i=1,2, \ldots, n \\
\omega_{i}^{-}+\sum_{j=1, j \neq i}^{n} \omega_{j}^{+} \geq 1, \quad i=1,2, \ldots, n \\
0<\omega_{i}^{-} \leq \omega_{i}^{+} \leq 1 \quad i=1,2, \ldots, n,
\end{array}\right.
\end{aligned}
$$

where the constraints are the normalization conditions of the $[0,1]$-valued interval weight vector $\bar{\omega}$ corresponding to (7), and $\omega_{i}^{-}(i=1,2, \ldots, n)$ and $\omega_{i}^{+}(i=1,2, \ldots, n)$ are decision variables.

Since $r_{i j}^{-}+r_{j i}^{+}=1$ and $r_{i j}^{+}+r_{j i}^{-}=1$ for all $i, j=1,2, \ldots, n$, one can obtain

$$
\begin{aligned}
\frac{\left(r_{j i}^{-} \omega_{i}^{+}-r_{i j}^{+} \omega_{j}^{-}\right)^{2}}{\left(r_{j i}^{-}\right)^{2}+\left(r_{i j}^{+}\right)^{2}} & =\frac{\left(r_{i j}^{+} \omega_{j}^{-}-r_{j i}^{-} \omega_{i}^{+}\right)^{2}}{\left(r_{i j}^{+}\right)^{2}+\left(r_{j i}^{-}\right)^{2}}, \quad i \neq j \\
\frac{\left(r_{j i}^{+} \omega_{i}^{-}-r_{i j}^{-} \omega_{j}^{+}\right)^{2}}{\left(r_{j i}^{+}\right)^{2}+\left(r_{i j}^{-}\right)^{2}} & =\frac{\left(r_{i j}^{-} \omega_{j}^{+}-r_{j i}^{+} \omega_{i}^{-}\right)^{2}}{\left(r_{i j}^{-}\right)^{2}+\left(r_{j i}^{+}\right)^{2}}, \quad i \neq j \\
\left(r_{i j}^{-}\right)^{2}+\left(r_{j i}^{+}\right)^{2} & =2\left(r_{i j}^{-}\right)^{2}-2 r_{i j}^{-}+1, \\
\left(r_{i j}^{+}\right)^{2}+\left(r_{j i}^{-}\right)^{2} & =2\left(r_{i j}^{+}\right)^{2}-2 r_{i j}^{+}+1 .
\end{aligned}
$$

Therefore, solutions to model (13) are found by solving the following optimization model:

$$
\begin{aligned}
& \min \quad J=\sum_{i=1}^{n-1} \sum_{j=i+1}^{n}\left(\frac{\left(r_{i j}^{-} \omega_{j}^{+}-\left(1-r_{i j}^{-}\right) \omega_{i}^{-}\right)^{2}}{2\left(r_{i j}^{-}\right)^{2}-2 r_{i j}^{-}+1}+\frac{\left(r_{i j}^{+} \omega_{j}^{-}-\left(1-r_{i j}^{+}\right) \omega_{i}^{+}\right)^{2}}{2\left(r_{i j}^{+}\right)^{2}-2 r_{i j}^{+}+1}\right) \\
& \text { s.t. }\left\{\begin{array}{l}
\omega_{i}^{+}+\sum_{j=1, j \neq i}^{n} \omega_{j}^{-} \leq 1, \\
\omega_{i}^{-}+\sum_{j=1, j \neq i}^{n} \omega_{j}^{+} \geq 1, \\
i=1,2, \ldots, n \\
0<\omega_{i}^{-} \leq \omega_{i}^{+} \leq 1, \quad i=1,2, \ldots, n .
\end{array}\right.
\end{aligned}
$$

Solving (15), one gets a normalized $[0,1]$-valued interval weight vector expressed as $\bar{\omega}^{*}=\left(\bar{\omega}_{1}^{*}, \bar{\omega}_{2}^{*}, \ldots, \bar{\omega}_{n}^{*}\right)^{T}=\left(\left[\omega_{1}^{-*}\right.\right.$, $\left.\left.\omega_{1}^{+*}\right],\left[\omega_{2}^{-*}, \omega_{2}^{+*}\right], \ldots,\left[\omega_{n}^{-*}, \omega_{n}^{+*}\right]\right)^{T}$.
Substituting $\bar{\omega}^{*}$ into (6), we obtain a multiplicatively consistent IFPR as

$$
\bar{R}^{*}=\left(\bar{r}_{i j}^{*}\right)_{n \times n}=\left(\left[r_{i j}^{-*}, r_{i j}^{+*}\right]\right)_{n \times n},
$$


TABLE 1: A comparative study for the IFPR $\bar{R}$.

\begin{tabular}{|c|c|c|c|}
\hline Model & Reference & Priority weight vector $\left(\bar{\omega}_{1}, \bar{\omega}_{2}, \bar{\omega}_{3}, \bar{\omega}_{4}\right)^{T}$ & Ranking \\
\hline $\begin{array}{l}(\mathrm{M}-8) \text { and } \\
(\mathrm{M}-9)\end{array}$ & $\begin{array}{c}\mathrm{Xu} \text { and Chen } \\
{[11]}\end{array}$ & $\begin{array}{l}([0.1568,0.3138],[0.1760,0.3592] \\
[0.2927,0.4934],[0.0847,0.1822])^{T}\end{array}$ & $x_{3} \stackrel{82.68 \%}{\succ} x_{2} \stackrel{59.49 \%}{\succ} x_{1} \stackrel{90.02 \%}{\succ} x_{4}$ \\
\hline $\begin{array}{l}\text { Equations }(22) \\
\text { and }(23)\end{array}$ & Genç et al. [12] & $\begin{array}{l}([0.1568,0.3138],[0.1760,0.3592], \\
[0.2927,0.4934],[0.0847,0.1822])^{T}\end{array}$ & $x_{3} \stackrel{82.68 \%}{\succ} x_{2} \stackrel{59.49 \%}{\succ} x_{1} \stackrel{90.02 \%}{\succ} x_{4}$ \\
\hline $\begin{array}{l}\text { Algorithm } 3 \\
\alpha=\beta=25 \\
\varepsilon=0.001\end{array}$ & Lan et al. [13] & $\begin{array}{c}([0.1575,0.3138],[0.1760,0.3580] \\
[0.2927,0.4934],[0.0847,0.1822])^{T}\end{array}$ & $x_{3} \stackrel{82.93 \%}{\succ} x_{2} \stackrel{59.26 \%}{\succ} x_{1} \stackrel{90.26 \%}{\succ} x_{4}$ \\
\hline $\begin{array}{l}\text { (MOD5) } \\
p=1,2,3,5\end{array}$ & $\mathrm{Xia}$ and $\mathrm{Xu}[14]$ & $\begin{array}{l}(0.2157,0.1989,0.4227,0.1627)^{T}, p=1 \\
(0.2310,0.2495,0.3848,0.1346)^{T}, p=2 \\
(0.2316,0.2496,0.3828,0.1360)^{T}, p=3 \\
(0.2329,0.2468,0.3791,0.1412)^{T}, p=5\end{array}$ & $\begin{array}{c}x_{3} \stackrel{100 \%}{\succ} x_{1} \stackrel{100 \%}{\succ} x_{2} \stackrel{100 \%}{\succ} x_{4}, p=1 \\
x_{3} \stackrel{100 \%}{>} x_{2} \stackrel{100 \%}{>} x_{1} \stackrel{100 \%}{\succ} x_{4}, p=2,3,5\end{array}$ \\
\hline Equation (15) & This paper & $\begin{array}{l}([0.1662,0.2677],[0.1706,0.3158] \\
[0.3012,0.4192],[0.0945,0.1425])^{T}\end{array}$ & $x_{3} \stackrel{94.45 \%}{\succ} x_{2} \stackrel{60.64 \%}{\succ} x_{1} \stackrel{100 \%}{\succ} x_{4}$ \\
\hline
\end{tabular}

where

$$
\left[r_{i j}^{-*}, r_{i j}^{+*}\right]= \begin{cases}{[0.5,0.5]} & i=j \\ {\left[\frac{\omega_{i}^{-*}}{\omega_{i}^{-*}+\omega_{j}^{+*}}, \frac{\omega_{i}^{+*}}{\omega_{j}^{-*}+\omega_{i}^{+*}}\right]} & i \neq j .\end{cases}
$$

If $J^{*}=0$, where $J^{*}$ is the optimal objective value of (15), then all of the DM's pairwise judgments in $\bar{R}$ are expressed as (17). It follows that $\bar{R}$ is the same as $\bar{R}^{*}$. Thus, $\bar{R}$ is a multiplicatively consistent IFPR.

Example 4. We discuss an MCDM problem concerning four decision alternatives $x_{1}, x_{2}, x_{3}$, and $x_{4}$. Denote the alternative set by $X=\left\{x_{1}, x_{2}, x_{3}, x_{4}\right\}$. A DM compares each pair of alternatives on $X$ and yields the following IFPR, which has been examined by Lan et al. [13]:

$$
\begin{aligned}
\bar{R} & =\left(\bar{r}_{i j}\right)_{4 \times 4}=\left(\left[r_{i j}^{-}, r_{i j}^{+}\right]\right)_{4 \times 4} \\
& =\left[\begin{array}{cccc}
{[0.5,0.5]} & {[0.36,0.66]} & {[0.26,0.45]} & {[0.57,0.72]} \\
{[0.34,0.63]} & {[0.5,0.5]} & {[0.32,0.52]} & {[0.55,0.77]} \\
{[0.55,0.74]} & {[0.48,0.68]} & {[0.5,0.5]} & {[0.66,0.83]} \\
{[0.28,0.43]} & {[0.23,0.45]} & {[0.17,0.34]} & {[0.5,0.5]}
\end{array}\right] .
\end{aligned}
$$

$$
\bar{R}^{*}=\left[\begin{array}{cccc}
{[0.5,0.5]} & {[0.3448,0.6108]} & {[0.2839,0.4706]} & {[0.5384,0.7391]} \\
{[0.3892,0.6552]} & {[0.5,0.5]} & {[0.2893,0.5118]} & {[0.5449,0.7697]} \\
{[0.5294,0.7161]} & {[0.4882,0.7107]} & {[0.5,0.5]} & {[0.6788,0.8160]} \\
{[0.2609,0.4616]} & {[0.2303,0.4551]} & {[0.1840,0.3212]} & {[0.5,0.5]}
\end{array}\right] .
$$

Next, four different approaches proposed by Xu and Chen [11], Genç et al. [12], Lan et al. [13], and Xia and Xu [14] are applied to the same IFPR $\bar{R}$ to derive priority weights that are summarized in Table 1.
Solving model (15) by the Optimization Modelling Software Lingo 11, one can obtain the following optimal $[0,1]$ valued interval weight vector:

$$
\begin{gathered}
\bar{\omega}^{*}=\left(\bar{\omega}_{1}^{*}, \bar{\omega}_{2}^{*}, \bar{\omega}_{3}^{*}, \bar{\omega}_{4}^{*}\right)^{T}=([0.1662,0.2677], \\
{[0.1706,0.3158],[0.3012,0.4192],} \\
[0.0945,0.1425])^{T} .
\end{gathered}
$$

By (8), the matrix of the possibility degree is determined as

$$
P=\left[\begin{array}{cccc}
0.5 & 0.3936 & 0 & 1 \\
0.6064 & 0.5 & 0.0555 & 1 \\
1 & 0.9445 & 0.5 & 1 \\
0 & 0 & 0 & 0.5
\end{array}\right]
$$

Summing all of elements in each line of $P$, we obtain $\rho_{1}=$ 1.8936, $\rho_{2}=2.1619, \rho_{3}=3.4445$, and $\rho_{4}=0.5$. As $\rho_{3}>\rho_{2}>$ $\rho_{1}>\rho_{4}$, the four alternatives are ranked as $x_{3} \stackrel{94.45 \%}{>} x_{2} \stackrel{60.64 \%}{>}$ $x_{1} \stackrel{100 \%}{>} x_{4}$

By (17), the corresponding multiplicatively consistent IFPR is determined as

Table 1 demonstrates that the ranking orders are nearly consistent based on the five different models. However, the values of the possibility degree of the obtained $[0,1]$-valued interval weights in this paper differ from the results derived 
from the other methods, which is due to the fact that the approaches adopt different consistency constraints for IFPRs. The transitivity conditions in [11-14] are all based on the feasible-region method; thus, $\bar{R}$ is judged to be a consistent IFPR. One can verify that $\bar{R}$ is not multiplicatively consistent under Definition 3. On the other hand, Xia and Xu's method [14] can only generate crisp priority weight vectors and yields distinct rankings under different parameter values for this particular IFPR. Lan et al.'s method [13] has to select appropriate parameters $\alpha$ and $\beta$, which seems difficult and complex.

\section{Geometric Least Square Models for Group Decision Making with IFPRs}

4.1. Derivation of Interval Weights Based on Individual IFPRs with Known Importance Weights. In the real-world situations, a decision is often made by a group of DMs. Suppose that an individual IFPR $\bar{R}_{k}=\left(\bar{r}_{i j k}\right)_{n \times n}=\left(\left[r_{i j k}^{-}, r_{i j k}^{+}\right]\right)_{n \times n}$ is furnished by the DM $d_{k}(k=1,2, \ldots, m)$ to express his/her preferences on the alternative set $X$. Let $D=\left\{d_{1}, d_{2}, \ldots, d_{m}\right\}$ be a set of $m$ DMs, and let $\lambda=\left(\lambda_{1}, \lambda_{2}, \ldots, \lambda_{m}\right)^{T}$ be an importance weight vector of $m$ DMs or a relative weight vector of IFPRs $\bar{R}_{k}(k=1,2, \ldots, m)$, which is known and satisfies $\sum_{k=1}^{m} \lambda_{k}=1$ and $\lambda_{k} \geq 0$ for all $k=1,2, \ldots, m$.

As different DMs generally have different subjective preferences or pair-wise judgments, it is nearly impossible to seek a common intersection point for planes $r_{i j k}^{-} \omega_{j}^{+}-r_{j i k}^{+} \omega_{i}^{-}=0$ and $r_{i j k}^{+} \omega_{j}^{-}-r_{j i k}^{-} \omega_{i}^{+}=0(i \neq j=1,2, \ldots, n, k=1,2, \ldots, m)$. In order to generate a unified $[0,1]$-valued interval weight vector for all individual IFPRs, the distances from a point to the planes are introduced as follows:

$$
\begin{aligned}
d_{i j k}^{(1)}= & \frac{\left|r_{i j k}^{-} \omega_{j}^{+}-r_{j i k}^{+} \omega_{i}^{-}\right|}{\sqrt{\left(r_{i j k}^{-}\right)^{2}+\left(r_{j i k}^{+}\right)^{2}}}, \\
d_{i j k}^{(2)}= & \frac{\left|r_{i j k}^{+} \omega_{j}^{-}-r_{j i k}^{-} \omega_{i}^{+}\right|}{\sqrt{\left(r_{i j k}^{+}\right)^{2}+\left(r_{j i k}^{-}\right)^{2}}} \\
& \forall i \neq j=1,2, \ldots, n, k=1,2, \ldots, m,
\end{aligned}
$$

where $d_{i j k}^{(1)}$ and $d_{i j k}^{(2)}$ denote the distances from the $2 n$-space point $\left(\omega_{1}^{-}, \omega_{1}^{+}, \omega_{2}^{-}, \omega_{2}^{+}, \ldots, \omega_{n}^{-}, \omega_{n}^{+}\right)^{T}$ to the planes $r_{i j k}^{-} \omega_{j}^{+}-$ $r_{j i k}^{+} \omega_{i}^{-}=0$ and $r_{i j k}^{+} \omega_{j}^{-}-r_{j i k}^{-} \omega_{i}^{+}=0$, respectively.

Once again, the smaller the sum of the values of the distances $d_{i j k}^{(1)}$ and $d_{i j k}^{(2)}$, the better the IFPR $\bar{R}_{k}$ from the viewpoint of the multiplicative consistency. As different IFPRs $\bar{R}_{k}(k=$ $1,2, \ldots, m)$ have different importance weights, a reasonable priority weight vector will be obtained by minimizing the weighted sum of these distances. Therefore, the following geometric least square model is established to derive a group $[0,1]$-valued interval weight vector directly from individual IFPRs:

$$
\begin{aligned}
& \min J=\sum_{k=1}^{m} \sum_{i=1}^{n} \sum_{j \neq i, j=1}^{n} \lambda_{k}\left(\frac{\left(r_{i j k}^{-} \omega_{j}^{+}-r_{j i k}^{+} \omega_{i}^{-}\right)^{2}}{\left(r_{i j k}^{-}\right)^{2}+\left(r_{j i k}^{+}\right)^{2}}+\frac{\left(r_{i j k}^{+} \omega_{j}^{-}-r_{j i k}^{-} \omega_{i}^{+}\right)^{2}}{\left(r_{i j k}^{+}\right)^{2}+\left(r_{j i k}^{-}\right)^{2}}\right) \\
& \text { s.t. }\left\{\begin{array}{l}
\omega_{i}^{+}+\sum_{j=1, j \neq i}^{n} \omega_{j}^{-} \leq 1, \quad i=1,2, \ldots, n \\
\omega_{i}^{-}+\sum_{j=1, j \neq i}^{n} \omega_{j}^{+} \geq 1, \quad i=1,2, \ldots, n \\
0<\omega_{i}^{-} \leq \omega_{i}^{+} \leq 1 \quad i=1,2, \ldots, n .
\end{array}\right.
\end{aligned}
$$

As $r_{i j k}^{-}+r_{j i k}^{+}=1$ and $r_{i j k}^{+}+r_{j i k}^{-}=1$ for all $i, j=$ $1,2, \ldots, n, k=1,2, \ldots, m$, we have

$$
\begin{aligned}
\frac{\left(r_{j i k}^{-} \omega_{i}^{+}-r_{i j k}^{+} \omega_{j}^{-}\right)^{2}}{\left(r_{j i k}^{-}\right)^{2}+\left(r_{i j k}^{+}\right)^{2}}=\frac{\left(r_{i j k}^{+} \omega_{j}^{-}-r_{j i k}^{-} \omega_{i}^{+}\right)^{2}}{\left(r_{i j k}^{+}\right)^{2}+\left(r_{j i k}^{-}\right)^{2}}, \\
i \neq j=1,2, \ldots, n, k=1,2, \ldots, m, \\
\frac{\left(r_{j i k}^{+} \omega_{i}^{-}-r_{i j k}^{-} \omega_{j}^{+}\right)^{2}}{\left(r_{j i k}^{+}\right)^{2}+\left(r_{i j k}^{-}\right)^{2}}=\frac{\left(r_{i j k}^{-} \omega_{j}^{+}-r_{j i k}^{+} \omega_{i}^{-}\right)^{2}}{\left(r_{i j k}^{-}\right)^{2}+\left(r_{j i k}^{+}\right)^{2}},
\end{aligned}
$$$$
i \neq j=1,2, \ldots, n, k=1,2, \ldots, m
$$

$$
\begin{aligned}
\left(r_{i j k}^{-}\right)^{2}+\left(r_{j i k}^{+}\right)^{2} & =2\left(r_{i j k}^{-}\right)^{2}-2 r_{i j k}^{-}+1, \\
i & \neq j=1,2, \ldots, n, k=1,2, \ldots, m, \\
\left(r_{i j k}^{+}\right)^{2}+\left(r_{j i k}^{-}\right)^{2} & =2\left(r_{i j k}^{+}\right)^{2}-2 r_{i j k}^{+}+1, \\
i & \neq j=1,2, \ldots, n, k=1,2, \ldots, m .
\end{aligned}
$$


Thus, solutions to model (23) are determined by solving the following geometric least square model:

$$
\begin{aligned}
& \min \quad J=\sum_{k=1}^{m} \sum_{i=1}^{n-1} \sum_{j=i+1}^{n} \lambda_{k}\left(\frac{\left(r_{i j k}^{-} \omega_{j}^{+}-\left(1-r_{i j k}^{-}\right) \omega_{i}^{-}\right)^{2}}{2\left(r_{i j k}^{-}\right)^{2}-2 r_{i j k}^{-}+1}+\frac{\left(r_{i j k}^{+} \omega_{j}^{-}-\left(1-r_{i j k}^{+}\right) \omega_{i}^{+}\right)^{2}}{2\left(r_{i j k}^{+}\right)^{2}-2 r_{i j k}^{+}+1}\right) \\
& \text { s.t. }\left\{\begin{array}{l}
\omega_{i}^{+}+\sum_{j=1, j \neq i}^{n} \omega_{j}^{-} \leq 1, \quad i=1,2, \ldots, n \\
\omega_{i}^{-}+\sum_{j=1, j \neq i}^{n} \omega_{j}^{+} \geq 1, \quad i=1,2, \ldots, n \\
0<\omega_{i}^{-} \leq \omega_{i}^{+} \leq 1 \quad i=1,2, \ldots, n .
\end{array}\right.
\end{aligned}
$$

Solving this model, a group $[0,1]$-valued interval weight vector is determined as

$$
\begin{gathered}
\bar{\omega}^{* G}=\left(\bar{\omega}_{1}^{* G}, \bar{\omega}_{2}^{* G}, \ldots, \bar{\omega}_{n}^{* G}\right)^{T}=\left(\left[\omega_{1}^{-* G}, \omega_{1}^{+* G}\right],\right. \\
\left.\left[\omega_{2}^{-* G}, \omega_{2}^{+* G}\right], \ldots,\left[\omega_{n}^{-* G}, \omega_{n}^{+* G}\right]\right)^{T} .
\end{gathered}
$$

4.2. Determination of Importance Weights of Individual IFPRs. Models (23) and (25) are developed by assuming the importance weights of DMs (or experts) or the relative weights of $m$ IFPRs to be known. However, in many real-world situations, it is difficult to directly assign importance weights to DMs or IFPRs because their importance depends on many factors such as expert's assessment level, DM's knowledge, and expertise related to the decision problem domain. In other words, the importance weights of DMs or the relative weights of $m$ IFPRs will have to be determined.

In group decision analysis, if $m$ IFPRs are the same, it is logical to assign their importance the same weights; that is, $\lambda_{k}=1 / m$ for all $=1,2, \ldots, m$. In this case, model $(25)$ is reduced to (15). If the IFPR $\bar{R}_{k}$ is much different from the others, its importance weight should be small and the geometric mean of the difference ratios between $\bar{R}_{k}$ and the others is large. Conversely, if $\bar{R}_{k}$ is very similar to the others, its importance should be high and the geometric mean of the difference ratios between $\bar{R}_{k}$ and the others is small. In order to determine the relative weights of individual IFPRs, a geometric mean based difference ratio between any two IFPRs is introduced as follows.

Definition 5. Let $\bar{R}_{k}=\left(\bar{r}_{i j k}\right)_{n \times n}=\left(\left[r_{i j k}^{-}, r_{i j k}^{+}\right]\right)_{n \times n}$ and $\bar{R}_{l}=$ $\left(\bar{r}_{i j l}\right)_{n \times n}=\left(\left[r_{i j l}^{-}, r_{i j l}^{+}\right]\right)_{n \times n}$ be any two IFPRs; then, the difference ratio between $\bar{R}_{k}$ and $\bar{R}_{l}$ is defined as

$$
\begin{gathered}
\operatorname{GMBDR}\left(\bar{R}_{k}, \bar{R}_{l}\right)=\left(\prod_{i \neq j}\left(\frac{\max \left\{r_{i j k}^{-}, r_{i j l}^{-}\right\}}{\min \left\{r_{i j k}^{-}, r_{i j l}^{-}\right\}}\right)\right. \\
\left.\cdot\left(\frac{\max \left\{r_{i j k}^{+}, r_{i j l}^{+}\right\}}{\min \left\{r_{i j k}^{+}, r_{i j l}^{+}\right\}}\right)\right)^{1 / 2\left(n^{2}-n\right)} .
\end{gathered}
$$

Obviously, $\operatorname{GMBDR}\left(\bar{R}_{k}, \bar{R}_{l}\right) \geq 1$ and $\operatorname{GMBDR}\left(\bar{R}_{k}, \bar{R}_{l}\right)=$ $\operatorname{GMBDR}\left(\bar{R}_{l}, \bar{R}_{k}\right)$. If $\operatorname{GMBDR}\left(\bar{R}_{k}, \bar{R}_{l}\right)=1$, one has $\bar{R}_{k}=\bar{R}_{l}$. The larger the difference ratio $\operatorname{GMBDR}\left(\bar{R}_{k}, \bar{R}_{l}\right)$ is, the less similar the $\bar{R}_{k}$ is to $\bar{R}_{l}$.

In order to derive the relative weights of individual IFPRs, the geometric average difference ratio between one IFPR and the others is introduced as follows.

Definition 6. Let $\bar{R}_{k}=\left(\bar{r}_{i j k}\right)_{n \times n}=\left(\left[r_{i j k}^{-}, r_{i j k}^{+}\right]\right)_{n \times n}(k=1$, $2, \ldots, m)$ be individual IFPRs; then, the geometric average difference ratio between $\bar{R}_{k}$ and the others is defined as

$$
\operatorname{GMDR}\left(\bar{R}_{k}\right)=\left(\prod_{l=1, l \neq k}^{m} \operatorname{GMBDR}\left(\bar{R}_{k}, \bar{R}_{l}\right)\right)^{1 /(m-1)} .
$$

It is obvious that $\operatorname{GMDR}\left(\bar{R}_{k}\right) \geq 1$. The smaller the $\operatorname{GMDR}\left(\bar{R}_{k}\right)$, the more important the $\bar{R}_{k}$ among individual IFPRs. In particular, if $\operatorname{GMDR}\left(\bar{R}_{k}\right)=1$, one can obtain $\operatorname{GMDR}\left(\bar{R}_{l}\right)=1$ for all $l=1,2, \ldots, m$; that is, $m$ IFPRs are completely the same. Therefore, the following formula can be employed to determine the relative weight of $\bar{R}_{k}$ :

$$
\lambda_{k}=\frac{1 / \operatorname{GMDR}\left(\bar{R}_{k}\right)}{\sum_{k=1}^{m}\left(1 / \operatorname{GMDR}\left(\bar{R}_{k}\right)\right)}, \quad k=1,2, \ldots, m .
$$

Clearly, we have $\sum_{k=1}^{m} \lambda_{k}=1$ and $0<\lambda_{k} \leq 1$ for all $k=$ $1,2, \ldots, m$.

Based on the above analyses, we now develop an approach for deriving a group $[0,1]$-valued interval weight vector directly from individual IFPRs with unknown importance weights. The approach is described in the following steps.

Step 1. For a group decision making problem with an alternative set $X=\left\{x_{1}, x_{2}, \ldots, x_{n}\right\}$, let $D=\left\{d_{1}, d_{2}, \ldots, d_{m}\right\}$ be the set of $m$ DMs. The DMs $d_{k}(k=1,2, \ldots, m)$ furnish their preferences by means of IFPRs $\bar{R}_{k}=\left(\bar{r}_{i j k}\right)_{n \times n}=\left(\left[r_{i j k}^{-}\right.\right.$, $\left.\left.r_{i j k}^{+}\right]\right)_{n \times n}(k=1,2, \ldots, m)$, where the relative importance 
weights of $\bar{R}_{k}(k=1,2, \ldots, m)$ are unknown, and $0<r_{i j k}^{-} \leq$ $r_{i j k}^{+}<1$ for all $i, j=1,2, \ldots, n, k=1,2, \ldots, m$.

Step 2. Calculate the difference ratios between $\bar{R}_{k}$ and $\bar{R}_{l}(k \neq$ $l=1,2, \ldots, m)$ as per $(27)$.

Step 3. Utilize (28) to calculate the difference ratio between $\bar{R}_{k}$ and the others for all $k=1,2, \ldots, m$.

Step 4. Utilize (29) to obtain the relative weight of $\bar{R}_{k}$ for all $k=1,2, \ldots, m$.

Step 5. Determine a group $[0,1]$-valued interval weight vector $\bar{\omega}^{* G}=\left(\bar{\omega}_{1}^{* G}, \bar{\omega}_{2}^{* G}, \ldots, \bar{\omega}_{n}^{* G}\right)^{T}$ by solving model (25).

Step 6. Calculate the possibility degree $P\left(\bar{\omega}_{i}^{* G} \geq \bar{\omega}_{j}^{* G}\right)(i, j=$ $1,2, \ldots, n)$ as per (8).

Step 7. Construct the possibility degree matrix $P=\left(p_{i j}\right)_{n \times n}$, where $p_{i j}=P\left(\bar{\omega}_{i}^{* G} \geq \bar{\omega}_{j}^{* G}\right)$.

Step 8. Adding all values in each row of $P$, we obtain $\rho_{i}=$ $\sum_{j=1}^{n} p_{i j}(i=1,2, \ldots, n)$.

Step 9. As per the decreasing order of $\rho_{i}$, a ranking order for all decision alternatives is derived, and " $x_{i}$ being preferred to $x_{j}$ " is expressed as $x_{i} \stackrel{P\left(\bar{\omega}_{i}^{* G} \geq \bar{\omega}_{j}^{* G}\right)}{\geq} x_{j}$.

\section{An Application to the Enterprise Resource Planning Software Product Selection Problem}

This section applies the proposed approach in Section 4 to an enterprise resource planning (ERP) software product selection problem that concerns group decision making with a hierarchical structure.

The ERP system has an important impact on improving the productivity of the organizations. However, the implementation of an ERP system is often very expensive and complex. Therefore, selecting the best suitable ERP software product is a vital decision making problem of the organizations when they aim to buy a ready ERP system in the market. Many factors or criteria impact the ERP software product selection [17]. In this case study, the ERP software product selection is made by the following five critical evaluation criteria: functionality $\left(c_{1}\right)$, cost and customization $\left(c_{2}\right)$, reliability $\left(c_{3}\right)$, compatibility $\left(c_{4}\right)$, and market position and reputation $\left(c_{5}\right)$.

Although there are many potential ERP software products in the market, only five of them, denoted by $x_{1}, x_{2}, x_{3}, x_{4}$, and $x_{5}$, are identified as candidates. A committee consisting of three experts $\left(d_{1}, d_{2}\right.$, and $\left.d_{3}\right)$ is set up to evaluate the five ERP software products, and its objective is to select the best one based on the above criterion scheme. The hierarchy of this ERP software product selection problem is shown in Figure 1.

As the importance weights of the five criteria are to be determined, each expert $d_{k}(k=1,2,3)$ compares each pair of the criteria and provides his/her judgments by means of an $\operatorname{IFPR} \bar{R}_{k}^{C}=\left(\bar{r}_{i j k}^{C}\right)_{5 \times 5}=\left(\left[r_{i j k}^{-C}, r_{i j k}^{+C}\right]\right)_{5 \times 5}$. Consider

$$
\bar{R}_{1}^{C}=\left[\begin{array}{lllll}
{[0.50,0.50]} & {[0.70,0.80]} & {[0.65,0.75]} & {[0.45,0.60]} & {[0.60,0.75]} \\
{[0.20,0.30]} & {[0.50,0.50]} & {[0.55,0.60]} & {[0.55,0.65]} & {[0.70,0.80]} \\
{[0.25,0.35]} & {[0.40,0.45]} & {[0.50,0.50]} & {[0.70,0.80]} & {[0.55,0.65]} \\
{[0.40,0.55]} & {[0.35,0.45]} & {[0.20,0.30]} & {[0.50,0.50]} & {[0.60,0.65]} \\
{[0.25,0.40]} & {[0.20,0.30]} & {[0.35,0.45]} & {[0.35,0.40]} & {[0.50,0.50]}
\end{array}\right],
$$$$
\bar{R}_{2}^{C}=\left[\begin{array}{lllll}
{[0.50,0.50]} & {[0.60,0.75]} & {[0.75,0.85]} & {[0.60,0.70]} & {[0.70,0.80]} \\
{[0.25,0.40]} & {[0.50,0.50]} & {[0.55,0.75]} & {[0.60,0.70]} & {[0.65,0.75]} \\
{[0.15,0.25]} & {[0.25,0.45]} & {[0.50,0.50]} & {[0.55,0.60]} & {[0.60,0.70]} \\
{[0.30,0.40]} & {[0.30,0.40]} & {[0.40,0.45]} & {[0.50,0.50]} & {[0.55,0.75]} \\
{[0.20,0.30]} & {[0.25,0.35]} & {[0.30,0.40]} & {[0.25,0.45]} & {[0.50,0.50]}
\end{array}\right],
$$

$$
\widehat{R}_{3}^{C}=\left[\begin{array}{lllll}
{[0.50,0.50]} & {[0.50,0.70]} & {[0.55,0.65]} & {[0.60,0.70]} & {[0.55,0.70]} \\
{[0.30,0.50]} & {[0.50,0.50]} & {[0.50,0.70]} & {[0.65,0.80]} & {[0.60,0.70]} \\
{[0.35,0.45]} & {[0.30,0.50]} & {[0.50,0.50]} & {[0.60,0.70]} & {[0.60,0.70]} \\
{[0.30,0.40]} & {[0.20,0.35]} & {[0.30,0.40]} & {[0.50,0.50]} & {[0.55,0.65]} \\
{[0.30,0.45]} & {[0.30,0.40]} & {[0.30,0.40]} & {[0.35,0.45]} & {[0.50,0.50]}
\end{array}\right] .
$$




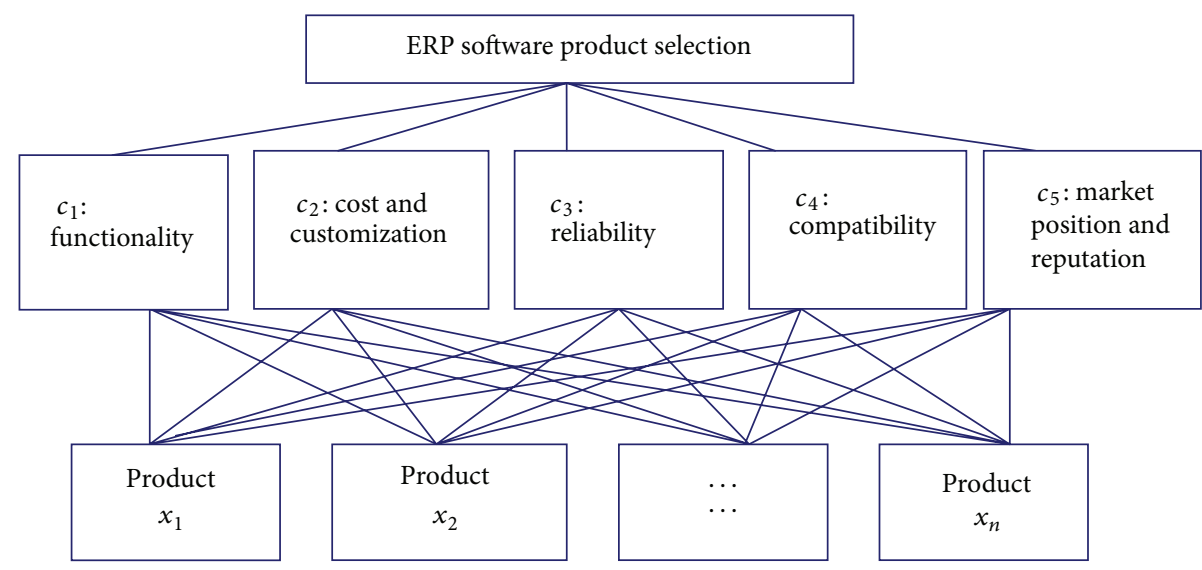

FIgURE 1: Decision hierarchy of an ERP software product selection problem.

On the other hand, the importance of the three experts is also unknown. Therefore, we need firstly to determine the relative weights of $\bar{R}_{k}^{C}(k=1,2,3)$.

By (27), one can obtain the difference ratios between $\bar{R}_{k}^{C}$ and $\bar{R}_{l}^{C}(k \neq l=1,2,3)$ as follows:

$$
\begin{aligned}
& \operatorname{GMBDR}\left(\bar{R}_{1}^{C}, \bar{R}_{2}^{C}\right)=\operatorname{GMBDR}\left(\bar{R}_{2}^{C}, \bar{R}_{1}^{C}\right)=1.2217, \\
& \operatorname{GMBDR}\left(\bar{R}_{1}^{C}, \bar{R}_{3}^{C}\right)=\operatorname{GMBDR}\left(\bar{R}_{3}^{C}, \bar{R}_{1}^{C}\right)=1.2280, \\
& \operatorname{GMBDR}\left(\bar{R}_{2}^{C}, \bar{R}_{3}^{C}\right)=\operatorname{GMBDR}\left(\bar{R}_{3}^{C}, \bar{R}_{2}^{C}\right)=1.1809 .
\end{aligned}
$$

As per (28), the difference ratios GMDR $\left(\bar{R}_{k}^{C}\right)(k=1,2,3)$ are calculated as $\operatorname{GMDR}\left(\bar{R}_{1}^{C}\right)=1.2248, \operatorname{GMDR}\left(\bar{R}_{2}^{C}\right)=$ 1.2011, and $\operatorname{GMDR}\left(\bar{R}_{3}^{C}\right)=1.4501$.

According to (29), the relative weights of $\bar{R}_{k}^{C}(k=1,2,3)$ are obtained as $\lambda_{1}=0.3491, \lambda_{2}=0.3560$, and $\lambda_{3}=0.2949$.

Next, substituting $\lambda_{k}$ and $\bar{R}_{k}^{C}(k=1,2,3)$ into (25) and solving this model, one gets normalized criterion weight vector $\bar{\omega}^{C}=\left(\left[\omega_{1}^{-C}, \omega_{1}^{+C}\right],\left[\omega_{2}^{-C}, \omega_{2}^{+C}\right], \ldots,\left[\omega_{5}^{-C}, \omega_{5}^{+C}\right]\right)^{T}=$ ([0.2789, 0.3424], [0.1783, 0.2418], [0.1380, 0.1883], [0.1228, $0.1613],[0.0887,0.1297])^{T}$.

Based on the criterion scheme, each expert $d_{k}(k=$ $1,2,3)$ compares each pair of the five ERP software products with respect to each criterion $c_{j}(j=1,2, \ldots, 5)$ and furnishes his/her judgments by an IFPR $\bar{R}_{k}^{c_{j}}=\left(\bar{r}_{i j k}^{c_{j}}\right)_{5 \times 5}=$ $\left(\left[r_{i j k}^{-c_{j}}, r_{i j k}^{+c_{j}}\right]\right)_{5 \times 5}$. Consider

$$
\begin{gathered}
\bar{R}_{1}^{c_{1}}=\left[\begin{array}{lllll}
{[0.50,0.50]} & {[0.55,0.65]} & {[0.40,0.55]} & {[0.45,0.60]} & {[0.35,0.45]} \\
{[0.35,0.45]} & {[0.50,0.50]} & {[0.35,0.45]} & {[0.30,0.40]} & {[0.25,0.35]} \\
{[0.45,0.60]} & {[0.55,0.65]} & {[0.50,0.50]} & {[0.45,0.60]} & {[0.40,0.55]} \\
{[0.40,0.55]} & {[0.60,0.70]} & {[0.40,0.55]} & {[0.50,0.50]} & {[0.35,0.50]} \\
{[0.55,0.65]} & {[0.65,0.75]} & {[0.45,0.60]} & {[0.50,0.65]} & {[0.50,0.50]}
\end{array}\right], \\
\bar{R}_{2}^{c_{1}}= \\
\bar{R}_{3}^{c_{1}}=\left[\begin{array}{lllll}
{[0.50,0.50]} & {[0.65,0.75]} & {[0.55,0.65]} & {[0.60,0.70]} & {[0.40,0.55]} \\
{[0.25,0.35]} & {[0.50,0.50]} & {[0.25,0.40]} & {[0.65,0.75]} & {[0.30,0.45]} \\
{[0.35,0.45]} & {[0.60,0.75]} & {[0.50,0.50]} & {[0.55,0.70]} & {[0.35,0.45]} \\
{[0.30,0.40]} & {[0.25,0.35]} & {[0.30,0.45]} & {[0.50,0.50]} & {[0.30,0.40]} \\
{[0.45,0.60]} & {[0.55,0.70]} & {[0.55,0.65]} & {[0.60,0.70]} & {[0.50,0.50]}
\end{array}\right], \\
{\left[\begin{array}{lllll}
{[0.50,0.50]} & {[0.35,0.45]} & {[0.30,0.55]} & {[0.25,0.45]} & {[0.35,0.60]} \\
{[0.55,0.70]} & {[0.25,0.35]} & {[0.50,0.50]} & {[0.35,0.65]} & {[0.25,0.45]} \\
{[0.55,0.75]} & {[0.60,0.70]} & {[0.35,0.65]} & {[0.50,0.50]} & {[0.45,0.75]} \\
{[0.40,0.65]} & {[0.70,0.90]} & {[0.55,0.75]} & {[0.25,0.55]} & {[0.50,0.50]}
\end{array}\right],}
\end{gathered}
$$




$$
\begin{aligned}
& \bar{R}_{1}^{\mathcal{C}_{2}}=\bar{R}_{2}^{\mathcal{C}_{2}}=\left[\begin{array}{lllll}
{[0.50,0.50]} & {[0.35,0.55]} & {[0.30,0.45]} & {[0.15,0.30]} & {[0.30,0.40]} \\
{[0.45,0.65]} & {[0.50,0.50]} & {[0.35,0.50]} & {[0.45,0.65]} & {[0.20,0.30]} \\
{[0.55,0.70]} & {[0.50,0.65]} & {[0.50,0.50]} & {[0.45,0.65]} & {[0.30,0.45]} \\
{[0.70,0.85]} & {[0.35,0.55]} & {[0.35,0.55]} & {[0.50,0.50]} & {[0.25,0.45]} \\
{[0.60,0.70]} & {[0.70,0.80]} & {[0.55,0.70]} & {[0.55,0.75]} & {[0.50,0.50]}
\end{array}\right], \\
& \bar{R}_{3}^{c_{2}}=\left[\begin{array}{lllll}
{[0.50,0.50]} & {[0.30,0.40]} & {[0.60,0.75]} & {[0.35,0.65]} & {[0.20,0.60]} \\
{[0.60,0.70]} & {[0.50,0.50]} & {[0.45,0.60]} & {[0.15,0.35]} & {[0.40,0.60]} \\
{[0.25,0.40]} & {[0.40,0.55]} & {[0.50,0.50]} & {[0.70,0.80]} & {[0.20,0.35]} \\
{[0.35,0.65]} & {[0.65,0.85]} & {[0.20,0.30]} & {[0.50,0.50]} & {[0.45,0.75]} \\
{[0.40,0.80]} & {[0.40,0.60]} & {[0.65,0.80]} & {[0.25,0.55]} & {[0.50,0.50]}
\end{array}\right], \\
& \bar{R}_{1}^{c_{3}}=\left[\begin{array}{lllll}
{[0.50,0.50]} & {[0.35,0.45]} & {[0.10,0.25]} & {[0.45,0.55]} & {[0.20,0.25]} \\
{[0.55,0.65]} & {[0.50,0.50]} & {[0.25,0.50]} & {[0.20,0.35]} & {[0.10,0.35]} \\
{[0.75,0.90]} & {[0.50,0.75]} & {[0.50,0.50]} & {[0.55,0.75]} & {[0.65,0.75]} \\
{[0.45,0.55]} & {[0.65,0.80]} & {[0.25,0.45]} & {[0.50,0.50]} & {[0.45,0.55]} \\
{[0.75,0.80]} & {[0.65,0.90]} & {[0.25,0.35]} & {[0.45,0.55]} & {[0.50,0.50]}
\end{array}\right], \\
& \bar{R}_{2}^{\mathcal{C}_{3}}=\bar{R}_{3}^{\mathcal{C}_{3}}=\left[\begin{array}{lllll}
{[0.50,0.50]} & {[0.65,0.75]} & {[0.10,0.30]} & {[0.35,0.45]} & {[0.35,0.55]} \\
{[0.25,0.35]} & {[0.50,0.50]} & {[0.25,0.30]} & {[0.25,0.45]} & {[0.30,0.45]} \\
{[0.70,0.90]} & {[0.70,0.75]} & {[0.50,0.50]} & {[0.50,0.55]} & {[0.45,0.65]} \\
{[0.55,0.65]} & {[0.55,0.75]} & {[0.45,0.50]} & {[0.50,0.50]} & {[0.35,0.45]} \\
{[0.45,0.65]} & {[0.55,0.70]} & {[0.35,0.55]} & {[0.55,0.65]} & {[0.50,0.50]}
\end{array}\right], \\
& \bar{R}_{1}^{c_{4}}=\bar{R}_{3}^{c_{4}}=\left[\begin{array}{lllll}
{[0.50,0.50]} & {[0.65,0.75]} & {[0.20,0.35]} & {[0.70,0.85]} & {[0.10,0.30]} \\
{[0.25,0.35]} & {[0.50,0.50]} & {[0.55,0.80]} & {[0.40,0.55]} & {[0.35,0.45]} \\
{[0.65,0.80]} & {[0.20,0.45]} & {[0.50,0.50]} & {[0.35,0.45]} & {[0.45,0.55]} \\
{[0.15,0.30]} & {[0.45,0.60]} & {[0.55,0.65]} & {[0.50,0.50]} & {[0.30,0.40]} \\
{[0.70,0.90]} & {[0.55,0.65]} & {[0.45,0.55]} & {[0.60,0.70]} & {[0.50,0.50]}
\end{array}\right], \\
& \bar{R}_{2}^{c_{4}}=\left[\begin{array}{lllll}
{[0.50,0.50]} & {[0.35,0.65]} & {[0.45,0.70]} & {[0.40,0.65]} & {[0.30,0.40]} \\
{[0.35,0.65]} & {[0.50,0.50]} & {[0.35,0.45]} & {[0.45,0.65]} & {[0.45,0.60]} \\
{[0.30,0.55]} & {[0.55,0.65]} & {[0.50,0.50]} & {[0.60,0.70]} & {[0.30,0.50]} \\
{[0.35,0.60]} & {[0.35,0.55]} & {[0.30,0.40]} & {[0.50,0.50]} & {[0.45,0.60]} \\
{[0.60,0.70]} & {[0.40,0.55]} & {[0.50,0.80]} & {[0.40,0.55]} & {[0.50,0.50]}
\end{array}\right], \\
& \bar{R}_{1}^{c_{5}}=\bar{R}_{2}^{c_{5}}=\bar{R}_{3}^{c_{5}}=\left[\begin{array}{llllll}
{[0.50,0.50]} & {[0.60,0.70]} & {[0.40,0.45]} & {[0.55,0.65]} & {[0.50,0.60]} \\
{[0.30,0.40]} & {[0.50,0.50]} & {[0.35,0.45]} & {[0.55,0.60]} & {[0.45,0.50]} \\
{[0.55,0.60]} & {[0.55,0.65]} & {[0.50,0.50]} & {[0.75,0.85]} & {[0.60,0.70]} \\
{[0.35,0.45]} & {[0.40,0.45]} & {[0.15,0.25]} & {[0.50,0.50]} & {[0.35,0.45]} \\
{[0.40,0.50]} & {[0.50,0.55]} & {[0.30,0.40]} & {[0.55,0.65]} & {[0.50,0.50]}
\end{array}\right] .
\end{aligned}
$$

For each criterion $c_{j}(j=1,2, \ldots, 5)$, by using (27), (28), and (29), the difference ratios $\operatorname{GMDR}\left(\bar{R}_{k}^{c_{j}}\right)$ and the relative weights of $\bar{R}_{k}^{c_{j}}(k=1,2,3)$ are determined as shown in Table 2. 
TABLE 2: Difference ratios and relative weights for individual IFPRs.

\begin{tabular}{|c|c|c|c|c|c|c|c|c|c|c|}
\hline \multirow{2}{*}{$k$} & \multicolumn{2}{|c|}{$\bar{R}_{k}^{c_{1}}$} & \multicolumn{2}{|c|}{$\bar{R}_{k}^{c_{2}}$} & \multicolumn{2}{|c|}{$\bar{R}_{k}^{c_{3}}$} & \multicolumn{2}{|c|}{$\bar{R}_{k}^{c_{4}}$} & \multicolumn{2}{|c|}{$\bar{R}_{k}^{c_{5}}$} \\
\hline & GMDR & Weight & GMDR & Weight & GMDR & Weight & GMDR & Weight & GMDR & Weight \\
\hline 1 & 1.3051 & 0.3961 & 1.2542 & 0.3575 & 1.3571 & 0.3004 & 1.2362 & 0.3560 & 1 & $1 / 3$ \\
\hline 2 & 1.4341 & 0.3605 & 1.2542 & 0.3575 & 1.1649 & 0.3498 & 1.5281 & 0.2880 & 1 & $1 / 3$ \\
\hline 3 & 2.1244 & 0.2434 & 1.5729 & 0.2850 & 1.1649 & 0.3498 & 1.2362 & 0.3560 & 1 & $1 / 3$ \\
\hline
\end{tabular}

TABLE 3: Local $[0,1]$-valued interval weights and the aggregated interval weights.

\begin{tabular}{lccccccc}
\hline Candidate & $c_{1}$ & $c_{2}$ & $c_{3}$ & $c_{4}$ & $c_{5}$ & Aggregated interval weights \\
& {$[0.2789,0.3424]$} & {$[0.1783,0.2418]$} & {$[0.1380,0.1883]$} & {$[0.1228,0.1613]$} & {$[0.0887,0.1297]$} & \\
\hline$x_{1}$ & {$[0.1601,0.2272]$} & {$[0.0949,0.1552]$} & {$[0.0949,0.1436]$} & {$[0.1174,0.1790]$} & {$[0.2003,0.2384]$} & {$[0.1287,0.2608]$} \\
$x_{2}$ & {$[0.1099,0.1456]$} & {$[0.1232,0.1828]$} & {$[0.0818,0.1298]$} & {$[0.1376,0.1992]$} & {$[0.1419,0.1709]$} & {$[0.1145,0.1643]$} \\
$x_{3}$ & {$[0.1525,0.2196]$} & {$[0.1444,0.2192]$} & {$[0.2553,0.3247]$} & {$[0.1485,0.2101]$} & {$[0.2760,0.3141]$} & {$[0.1779,0.2504]$} \\
$x_{4}$ & {$[0.1344,0.1907]$} & {$[0.1318,0.2078]$} & {$[0.1719,0.2209]$} & {$[0.1222,0.1838]$} & {$[0.0970,0.1243]$} & {$[0.1321,0.1937]$} \\
$x_{5}$ & {$[0.2170,0.2840]$} & {$[0.2348,0.3108]$} & {$[0.1810,0.2504]$} & {$[0.2277,0.2893]$} & {$[0.1523,0.1905]$} & {$[0.2067,0.2775]$} \\
\hline
\end{tabular}

For each criterion $c_{j}(j=1,2, \ldots, 5)$, plugging $\bar{R}_{k}^{c_{j}}(k=$ $1,2,3)$ and their relative weights into (25) and solving this model, one can obtain a normalized $[0,1]$-valued interval weight vector for $x_{i}(i=1,2, \ldots, 5)$ with respect to the criterion $c_{j}$, denoted by $\left(\left[\omega_{1 j}^{-}, \omega_{1 j}^{+}\right],\left[\omega_{2 j}^{-}, \omega_{2 j}^{+}\right], \ldots,\left[\omega_{5 j}^{-}, \omega_{5 j}^{+}\right]\right)^{T}$, as listed in columns 1-5 in Table 3, where the first row gives the criterion weights $\bar{\omega}_{j}^{* C}(j=1,2, \ldots, 5)$ derived earlier.

Similar to the treatment in Wang and Li [15], the following linear programs given by Bryson and Mobolurin [18] are applied to aggregate local $[0,1]$-valued interval weights into the aggregated interval weights for $x_{i}(i=1,2, \ldots, 5)$. Consider

$$
\begin{array}{ll}
\min & \omega_{x_{i}}^{-}=\sum_{j=1}^{5} \omega_{i j}^{-} \omega_{j} \\
\text { s.t. } & \left\{\begin{array}{l}
\omega_{j}^{-C} \leq \omega_{j} \leq \omega_{j}^{+C}, \quad j=1,2, \ldots, 5 \\
\sum_{j=1}^{5} \omega_{j}=1,
\end{array}\right. \\
\max \quad \omega_{x_{i}}^{+}=\sum_{j=1}^{5} \omega_{i j}^{+} \omega_{j} \\
\text { s.t. }\left\{\begin{array}{l}
\omega_{j}^{-C} \leq \omega_{j} \leq \omega_{j}^{+C}, \quad j=1,2, \ldots, 5 \\
\sum_{j=1}^{5} \omega_{j}=1,
\end{array}\right.
\end{array}
$$

where $\omega_{j}$ (for $\left.j=1,2, \ldots, 5\right)$ are decision variables.

In (33), $\left[\omega_{j}^{-C}, \omega_{j}^{+C}\right]$ (for $\left.j=1,2, \ldots, 5\right)$ are the normalized $[0,1]$-valued interval weights of the five criteria, and $\left[\omega_{i j}^{-}, \omega_{i j}^{+}\right]$ (for $i=1,2, \ldots, 5$ ) are the normalized $[0,1]$-valued interval weights for the five alternatives over the criterion $c_{j}(j=$ $1,2, \ldots, 5)$. They are determined and shown in Table 3.

Solving (33) yields the aggregated $[0,1]$-valued interval weight $\bar{\omega}_{x_{i}}=\left[\omega_{x_{i}}^{-}, \omega_{x_{i}}^{+}\right]$for $x_{i}(i=1,2, \ldots, 5)$ as listed in the last column in Table 3 .
By (8), we obtain the possibility degree matrix as follows:

$$
P=\left[\begin{array}{ccccc}
0.5 & 0.8043 & 0.4052 & 0.6644 & 0.2666 \\
0.1957 & 0.5 & 0 & 0.2890 & 0 \\
0.5948 & 1 & 0.5 & 0.8822 & 0.3050 \\
0.3356 & 0.7110 & 0.1178 & 0.5 & 0 \\
0.7234 & 1 & 0.6950 & 1 & 0.5
\end{array}\right]
$$

Adding up all of elements in each row of $P$, we have $\rho_{1}=$ 2.6405, $\rho_{2}=0.9847, \rho_{3}=3.2820, \rho_{4}=1.6644$, and $\rho_{5}=$ 3.9184. As $\rho_{5}>\rho_{3}>\rho_{1}>\rho_{4}>\rho_{2}$, the five ERP software products are ranked as $x_{5} \stackrel{69.5 \%}{\succ} x_{3} \stackrel{59.48 \%}{>} x_{1} \stackrel{66.44 \%}{>} x_{4} \stackrel{71.1 \%}{>} x_{2}$.

\section{Conclusions}

Derivation of priority weights from IFPRs plays an important role for MCDM with interval fuzzy preference information. In this paper, we have analyzed the relationship among the normalized $[0,1]$-valued interval weights, multiplicatively consistent interval judgments, and planes. A geometric least square model has been developed for deriving $[0,1]$-valued interval weights from any IFPR and extended to generate a group $[0,1]$-valued interval weight vector directly from individual IFPRs, whose relative weights are assumed to be known. We have introduced the notion of the geometric average difference ratio between one IFPR and the others and applied it to determine the relative importance weights of individual IFPRs. A geometric least squares based approach has been put forward for group decision making with IFPRs. We have provided a numerical example and comparative analyses to illustrate the validity of the proposed models and presented a case study to show that the proposed framework is operational in practice.

In the future, we will focus on the ratio-based geometric similarity measure on IFPRs and its application to consensus models of group decision making. 


\section{Conflict of Interests}

The authors declare that there is no conflict of interests regarding the publication of this paper.

\section{Acknowledgments}

The research is supported by the Zhejiang Provincial Natural Science Foundation of China under Grant LY15G010004 and the National Natural Science Foundation of China under Grant 71271188.

\section{References}

[1] T. L. Saaty, The Analytic Hierarchy Process, McGraw-Hill, New York, NY, USA, 1980.

[2] F. Chiclana, E. Herrera-Viedma, F. Alonso, and S. Herrera, "Cardinal consistency of reciprocal preference relations: a characterization of multiplicative transitivity," IEEE Transactions on Fuzzy Systems, vol. 17, no. 1, pp. 14-23, 2009.

[3] M. Xia, Z. Xu, and J. Chen, "Algorithms for improving consistency or consensus of reciprocal [0,1]-valued preference relations," Fuzzy Sets and Systems, vol. 216, pp. 108-133, 2013.

[4] X. Liu, Y. Pan, Y. Xu, and S. Yu, "Least square completion and inconsistency repair methods for additively consistent fuzzy preference relations," Fuzzy Sets and Systems, vol. 198, pp. 1-19, 2012.

[5] R.-H. Chiu, L.-H. Lin, and S.-C. Ting, "Evaluation of green port factors and performance: a fuzzy AHP analysis," Mathematical Problems in Engineering, vol. 2014, Article ID 802976, 12 pages, 2014.

[6] C.-C. Chou and K.-W. Yu, "Application of a new hybrid fuzzy AHP model to the location choice," Mathematical Problems in Engineering, vol. 2013, Article ID 592138, 12 pages, 2013.

[7] Z. Xu, "On compatibility of interval fuzzy preference relations," Fuzzy Optimization and Decision Making, vol. 3, no. 3, pp. 217225, 2004.

[8] T. Entani and K. Sugihara, "Uncertainty index based interval assignment by interval AHP," European Journal of Operational Research, vol. 219, no. 2, pp. 379-385, 2012.

[9] P. Guo and Y. Wang, "Eliciting dual interval probabilities from interval comparison matrices," Information Sciences, vol. 190, pp. 17-26, 2012.

[10] K. Sugihara, H. Ishii, and H. Tanaka, "Interval priorities in AHP by interval regression analysis," European Journal of Operational Research, vol. 158, no. 3, pp. 745-754, 2004.

[11] Z. Xu and J. Chen, "Some models for deriving the priority weights from interval fuzzy preference relations," European Journal of Operational Research, vol. 184, no. 1, pp. 266-280, 2008.

[12] S. Genç, F. E. Boran, D. Akay, and Z. Xu, "Interval multiplicative transitivity for consistency, missing values and priority weights of interval fuzzy preference relations," Information Sciences, vol. 180, no. 24, pp. 4877-4891, 2010.

[13] J. Lan, M. Hu, X. Ye, and S. Sun, "Deriving interval weights from an interval multiplicative consistent fuzzy preference relation," Knowledge-Based Systems, vol. 26, pp. 128-134, 2012.

[14] M. Xia and Z. Xu, "Interval weight generation approaches for reciprocal relations," Applied Mathematical Modelling, vol. 38, no. 3, pp. 828-838, 2014.
[15] Z.-J. Wang and K. W. Li, "Goal programming approaches to deriving interval weights based on interval fuzzy preference relations," Information Sciences, vol. 193, pp. 180-198, 2012.

[16] T. Tanino, "Fuzzy preference orderings in group decision making," Fuzzy Sets and Systems, vol. 12, no. 2, pp. 117-131, 1984.

[17] T. Gürbüz, S. E. Alptekin, and G. I. Alptekin, "A hybrid MCDM methodology for ERP selection problem with interacting criteria," Decision Support Systems, vol. 54, no. 1, pp. 206-214, 2012.

[18] N. Bryson and A. Mobolurin, "An action learning evaluation procedure for multiple criteria decision making problems," European Journal of Operational Research, vol. 96, no. 2, pp. 379386, 1997. 


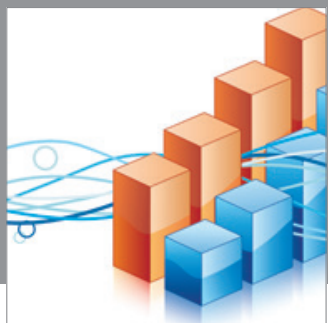

Advances in

Operations Research

mansans

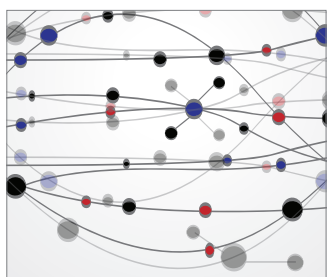

The Scientific World Journal
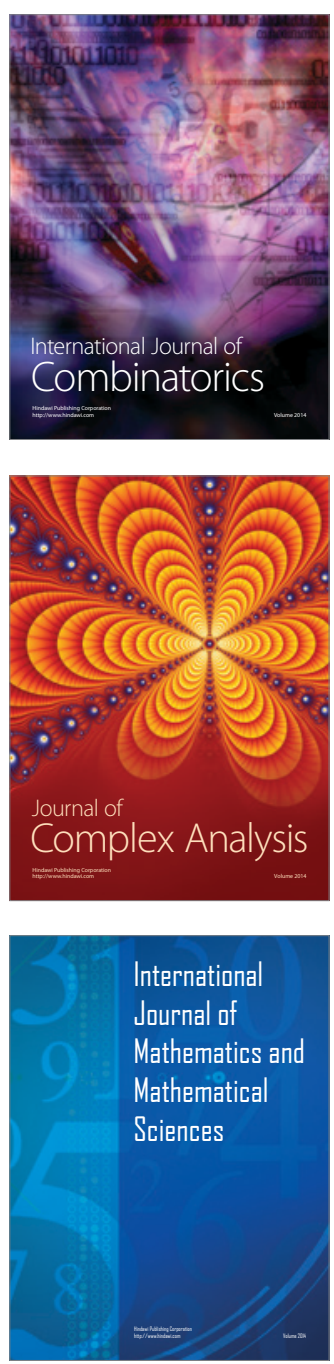
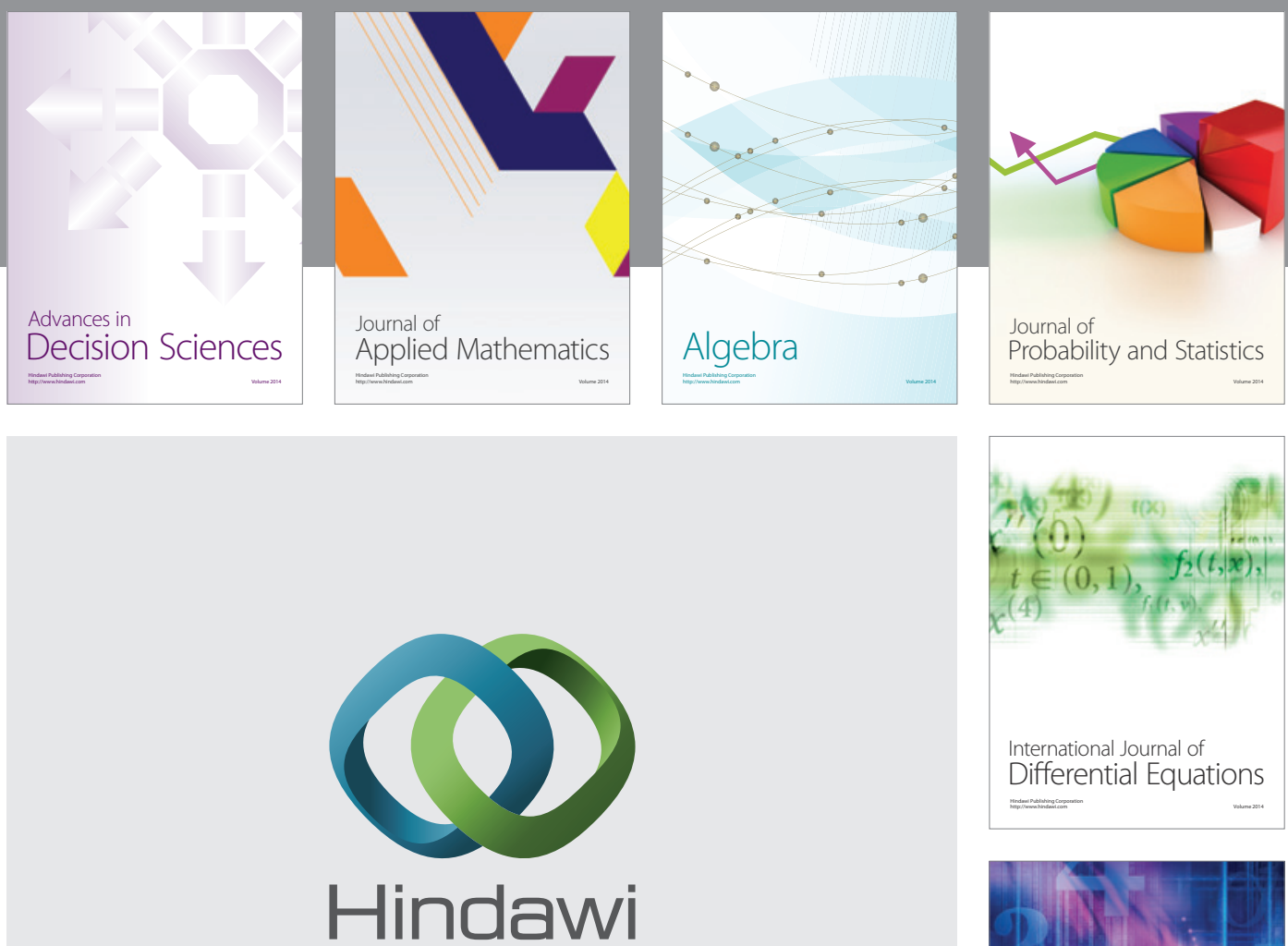

Submit your manuscripts at http://www.hindawi.com
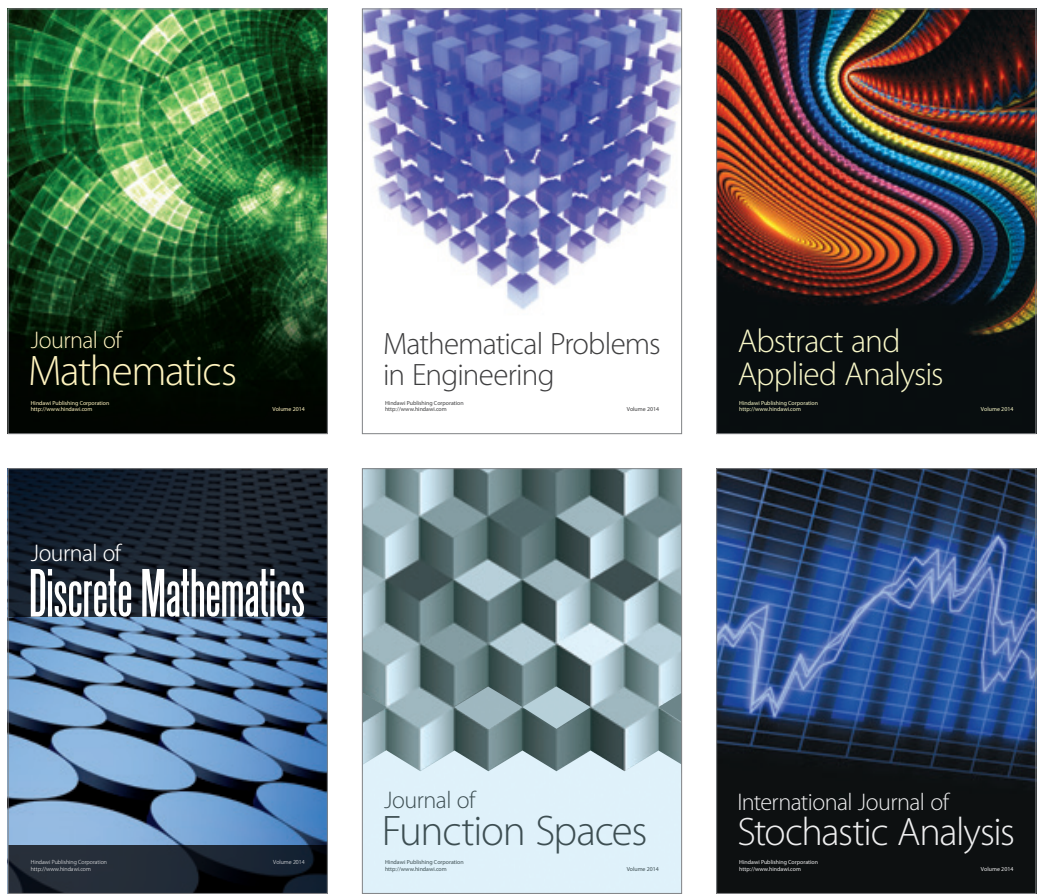

Journal of

Function Spaces

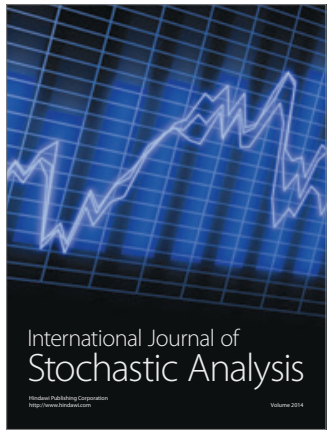

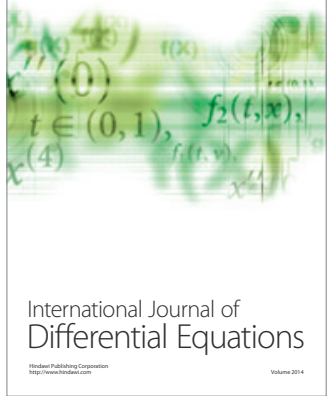
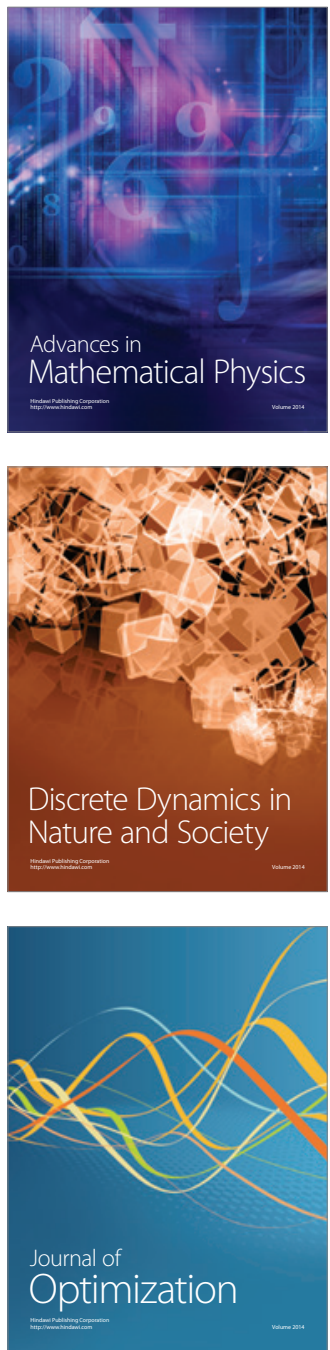